\title{
Collective Electronic Oscillator Method: Application to Conjugated Organic Molecules
}

\author{
Jin Yong Lee \\ Department of Chemistry. Chonnam National Lniversity, Gwangin 500-757, Korea \\ Received February 28, 2003
}

\begin{abstract}
The collective electronic oscillator (CEO) method was developed by Mukamel and collaborators. [Phs. Rev. Lett. 1992. 69, 65: Science 1997, 277. 781] Recently I have extended the CEO method to obtain the frequency dependent optical properties with all the contributing components. The brief introduction of the CEO fomalism and its recent applications to linear absorption and two-photon absorption (TPA) of conjugated organic molecules will be discussed. The size scaling of optical properties of polyenes and polyynes have studied by ab initio calculations. and this result is consistent with the coherence length of the time dependent densities to first $\left(\rho^{\prime 1)}\right)$ and second order $\left(\rho^{\prime 2}\right)$ in the electric field obtained from the CEO method.
\end{abstract}

Key Words : Collective electronic oscillator. Two-photon absorption

\section{Introduction}

Conjugated organic molecules or polymers are primary candidates for new organic optical materials with large nonlinear polarizabilities. ${ }^{1-3}$ Potential applications include electroluminescence, light emiting diodes. ultrafast switches, multiphoton microscopy. etc. ${ }^{46}$ Optical spectroscopy allows us to probe the dynamics of vibrations and electronic excitations of molecules. For the theoretical study to interprete the optical spectroscopic signals. the many-electron problem is required to be solved. Several approaches have been applied towards the computation of nonlinear optical susceptibilities. One of the most commonly used is based on Ward's pertubative sum-over-state (SOS) method expression. ${ }^{7-10}$ Due to its numerical nature, it is more successful in accounting for the experimentally deternined magnitudes rather than in providing guidelines for molecular engineering. For a better description of multiply excited configurations. a quantitative analysis has been proposed to identify those states which significantly' contribute to the SOS expansion, and applied to the quadratic and cubic polarizabilities of standard charge transfer in aromatic molecules. ${ }^{11.12}$ Various ways to extract the dominant excitestate contributions to the expansion and to relate these states to specific molecular features have been proposed. In particular. the two-state model is often successfully used to describe the quadratic optical polarizabilities where the charge transfer is crucial. ${ }^{13-15}$ Alternatively the finite-field method has been applied to evaluate the static hyperpolarizabilities of charge transfer sy'stems and is being routinely implemented in quantum chemistry packages. ${ }^{16}$ This method focuses on ground-state wasefunctions and unlike the SOS does not incorporate explicitly dipolar transitions between various states.

Consequently it cannot identify the excited states which contribute significantly to the nonlinear optical properties of interest

\footnotetext{
${ }^{*}$ E-mail jinyong achomam.ac.ks
}

Mukamel and collaborators have developed a more general collective electronic oscillators (CEO) method which is applicable for both off resonant and resonant responses. ${ }^{17-1.9}$ The $\mathrm{CEO}$ framework has been used to compute and interpret the off-resonant lyyperpolarizabilities of push-pull carotenoids."? It was further estended to frequency dependent resonant optical properties. ${ }^{21}$ Recently. a new synthetic family. with through-space charge transfer (4-(4-dihexy laminostyryl)-16(4-nitrostyryl)[2.2] paracyclophanes) have been synthesized."2 Their absorption spectra were measured and the off-resonant first lyperpolarizabilities were investigated using the collective electronic oscillator (CEO) approach ${ }^{33}$ with various throughspace charge transfer network. In this minireview, I introduce the CEO method and its application to investigate the nonlinear optical properties.

\section{CEO Method}

The CEO method uses molecular geometries as an input which usually optimized with quantum chemical methods or obtained from experimental X-ray diffraction or NMR data. The detailed CEO formalism is available in a recent review article by Tretiak and Mukanel. ${ }^{\text {" }}$ Here the basic procedure will be described briefly. Basically. CEO method solves the time dependent Hartree-Fock (TDHF) equation of motion which involves the diagonalization of the Liouville operator. which is efficiently performed using Krylov-space techniques such as IDSMA. ${ }^{24.25}$ Lanczos. ${ }^{26 i}$ and Davidson $\mathrm{s}^{27.28}$ algoritlums.

CEO starts with the Heisenberg equation of motion for $\rho_{n m}(t)=\left\langle c_{n}^{\dagger} c_{n}\right\rangle$ :

$$
i \frac{\partial \rho_{m m}}{\partial t}=\left\langle\left[c_{n}^{\dagger} c_{m}, H\right]\right\rangle .
$$

where

$$
\begin{aligned}
& H=\sum_{n i n \sigma} t_{m m} c_{m \sigma^{\prime}}^{\dagger} c_{n \sigma}-\sum_{m n+k\}}\langle n m \mid k l\rangle c_{m \sigma}^{*} c_{n \sigma^{\prime}}^{*} c_{k \sigma^{\prime}} c_{l \sigma} \\
& -\varepsilon(t) \sum_{m n \sigma} \mu_{n n} c_{m \sigma^{\prime}} c_{n \sigma^{\prime}} \sigma^{\prime}
\end{aligned}
$$


This equation is not closed since higher order products (two-electron density matrices) show up in the right-hand side as is common to many-body dynamics in classical and quantum mechanics. By a tnuncation procedure. a closed time-dependent Hartree-Fock equation is obtained for the single electron density matrix $\rho(t)$.

$$
i \frac{\partial \rho}{\partial t}=i \frac{\partial \delta \rho}{\partial t}=[F(\rho), \rho]-\varepsilon(t) \cdot[\mu, \rho]
$$

This set of equations can be solved in frequency or time domain. By restricting the number of equations to variables which contain only' M occupied-unoccupied orbital pairs. the algebra of electronic oscillators was developed. To that end. $\delta \rho(t)$ is decomposed into two components. particle-hole (interband) parts $(\xi)$ and particle-particle and hole-hole (intraband) parts $(T(\xi))$

$$
\delta \rho(t)=\xi(t)-T(\xi(t))
$$

Using the idempotent properties of density matrices. the intraband parts can be expanded in powers of interband parts

$$
T(\xi)=\frac{1}{2 !}[[\xi, \bar{\rho}], \xi]+\frac{1}{4 !}[[\xi, \bar{\rho}],[\xi, \bar{\rho}],[\xi, \bar{\rho}], \xi]+\ldots
$$

Thus, the TDHF equation is obtained for $\xi$

$$
i \frac{\partial \xi}{\partial t}-L \xi=R(\xi)-\varepsilon(t) \cdot[\mu, \bar{\rho}]
$$

where $L$ is a linear operator in Liouville spacegiven by

$$
L \xi=[F(\bar{\rho})]+[V(\xi), \bar{\rho}]
$$

and $\quad R(\xi)=[F(\xi), \xi+T(\xi)]+[F(T(\xi)), \bar{\rho}+\xi]$

$$
-\varepsilon(t) \cdot[\mu, \xi+T(\xi)] \text {. }
$$

Then. the TDHF equation can be solved by expanding the density matrix in powers of the external electric field

$$
\xi=\xi^{(1)}+\xi^{(3)}+\xi^{(3)}+\ldots, \quad T(\xi)=T^{(2)}(\xi)+T^{(3)}(\xi)+\ldots
$$

The time dependent polarization which determines the optical properties is given by

$$
P(t)=\operatorname{Tr}(\mu \rho(t))=\operatorname{Tr}(\mu \xi(t))-\operatorname{Tr}(\mu T(\xi(t))),
$$

and the polarization to $j$ th order in the external electric field $\varepsilon(t)$ is obtained by taking the expectation value of the dipole operator $\mu$ with respect to the time-dependent density matrix

$$
P^{i j !} t=T r\left(\mu \delta \rho^{l}(t)\right)
$$

Therefore we can obtain the following optical properties:

$$
\begin{aligned}
& \alpha^{i j}(\omega)=\sum_{\alpha} \frac{S_{\alpha} \mu_{-\alpha}^{l} \mu_{\alpha}^{j}}{\Omega_{\alpha \alpha}-\omega} . \\
& \beta^{3 k^{k}}\left(\omega_{1}, \omega_{2}\right)=-\sum_{\alpha \beta \gamma \gamma} \frac{V_{-\alpha_{1} \beta \gamma} S_{\alpha \alpha} S_{\beta} S_{\gamma} \mu_{\alpha}^{l} \mu_{-\beta}^{j} \mu_{-\gamma}^{k}}{\left(\Omega_{\alpha}-\omega_{1}-\omega_{2}\right)\left(\Omega_{\beta}-\omega_{1}\right)\left(\Omega_{\gamma}-\omega_{2}\right)} \\
&-\frac{1}{2} \sum_{\alpha \beta \beta} \frac{S_{\alpha \alpha} S_{\beta} \mu_{\alpha \alpha}^{i} \mu_{-\alpha \beta}^{j} \mu_{-\beta}^{k}}{\left(\Omega_{\alpha}-\omega_{1}-\omega_{2}\right)\left(\Omega_{\beta}-\omega_{2}\right)}
\end{aligned}
$$

$$
\begin{aligned}
& +\frac{1}{2} \sum_{\alpha \beta} \frac{S_{\alpha} S_{\beta} \mu_{\alpha \mu}^{2} \mu_{-\alpha \beta}^{l} \mu_{-\beta}^{k}}{\left(\Omega_{\alpha}-\omega_{1}-\omega_{2}\right)\left(\Omega_{\beta}-\omega_{1}\right)} \\
& +\frac{1}{2} \sum_{\alpha \beta} \frac{S_{\alpha \beta} S_{\beta} \mu_{\alpha \beta \beta}^{l} \mu_{-\alpha}^{l} \mu_{-\beta}^{k}}{\left(\Omega_{\alpha}-\omega_{1}\right)\left(\Omega_{\beta}-\omega_{2}\right)} .
\end{aligned}
$$

$$
\begin{aligned}
\gamma^{i j k l}\left(\omega_{1} \cdot \omega_{2} \cdot \omega_{3}\right) & =\frac{1}{6} \sum_{\omega_{1} \omega_{2} \omega_{3}}^{p e r m b}\left(\gamma_{I}^{j k l}+\gamma_{I J}^{j k l}+\gamma_{I I I}^{i j k l}+\gamma_{I V}^{j j k l}\right. \\
& \left.+\gamma_{l}^{j k l}+\gamma_{l I}^{j k l}+\gamma_{I I I}^{j k l}+\gamma_{i I I I}^{j k l}\right)
\end{aligned}
$$

$\gamma_{I}^{j k l}\left(\omega_{1}, \omega_{2}, \omega_{3}\right)=$

$$
\sum_{\alpha \beta \gamma \gamma} \frac{S_{\alpha \beta} S_{\beta} S_{\gamma} \mu_{\alpha}^{l} \mu_{-\alpha \beta \beta}^{l} \mu_{-\beta \gamma}^{k} \mu_{-\gamma}^{l}}{\left(\Omega_{\alpha}-\omega_{1}-\omega_{2}-\omega_{3}\right)\left(\Omega_{\beta}-\omega_{2}-\omega_{3}\right)\left(\Omega_{\gamma}-\omega_{2}\right)}
$$

$\gamma_{J j}^{i j k j}\left(\omega_{1}, \omega_{2}, \omega_{2}\right)=$

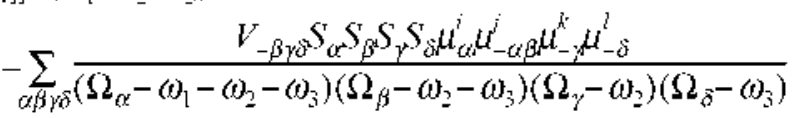

$$
\begin{aligned}
& \gamma_{H I}^{i j k l}\left(\omega_{1} . \omega_{2} \cdot \omega_{3}\right)= \\
& \quad \sum_{\alpha \beta \gamma} \frac{S_{\alpha} S_{\beta} S_{\gamma} \mu_{\alpha}^{2} \mu_{-\alpha \beta}^{l} \mu_{-\beta}^{k} \mu_{-\gamma}^{\prime}}{\left(\Omega_{\alpha}-\omega_{1}-\omega_{2}-\omega_{3}\right)\left(\Omega_{\beta}-\omega_{2}-\omega_{3}\right)\left(\Omega_{\gamma}-\omega_{3}\right)}
\end{aligned}
$$

$\gamma_{I V^{\prime}}^{i j k}\left(\omega_{1}, \omega_{2}, \omega_{3}\right)=$

$-2 \sum_{\alpha \beta \gamma \delta} \frac{V_{-\beta \gamma \delta} S_{\alpha} S_{\beta} S_{\gamma} S_{\delta} \mu_{\alpha}^{i} \mu_{-\alpha \beta}^{j} \mu_{-j}^{k} \mu_{-\delta}^{l}}{\left(\Omega_{\alpha}-\omega_{1}-\omega_{2}-\omega_{3}\right)\left(\Omega_{\beta}-\omega_{2}-\omega_{3}\right)\left(\Omega_{\gamma}-\omega_{1}\right)\left(\Omega_{\bar{\delta}}-\omega_{3}\right)}$

$\gamma_{l}^{i j k l}\left(\omega_{1} \cdot \omega_{-} . \omega_{3}\right)=2 \sum_{\alpha \beta \gamma \delta \eta}$

$\frac{V_{-\alpha \beta \gamma} V_{-\gamma \delta \eta} S_{\alpha} S_{\beta} S_{\gamma} S_{\delta} S_{\eta} \mu_{\alpha \mu}^{i} \mu_{-\beta}^{i} \mu_{-\delta}^{k} \mu_{-\eta}^{l}}{\left(\Omega_{\alpha}-\omega_{1}-\omega_{2}-\omega_{3}\right)\left(\Omega_{\beta}-\omega_{1}\right)\left(\Omega_{\gamma}-\omega_{2}-\omega_{3}\right)\left(\Omega_{\delta}-\omega_{2}\right)\left(\Omega_{\eta}-\omega_{3}\right)}$

$$
\begin{aligned}
& \gamma_{17}^{i j k l}\left(\omega_{1}, \omega_{2}, \omega_{2}\right)= \\
& -\sum_{\alpha \beta \gamma \delta \delta} \frac{V_{-\alpha \beta \gamma \delta} S_{\alpha \alpha} S_{\beta} S_{\gamma} S_{j i} \mu_{\alpha}^{i} \mu_{-\beta}^{j} \mu_{-\gamma}^{k} \mu_{-\delta}^{l}}{\left(\Omega_{\alpha}-\omega_{1}-\omega_{2}-\omega_{3}\right)\left(\Omega_{\beta}-\omega_{1}\right)\left(\Omega_{\gamma}-\omega_{2}\right)\left(\Omega_{\delta}-\omega_{3}\right)}
\end{aligned}
$$

$$
\gamma_{17 I}^{\eta j l}\left(\omega_{1} . \omega_{2} . \omega_{3}\right)=\sum_{\alpha \beta \gamma} \frac{S_{\alpha} S_{\beta} S_{\gamma} \mu_{\alpha \beta \beta}^{l} \mu_{-\alpha \beta}^{l} \mu_{-\beta \gamma}^{k} \mu_{-\gamma}^{l}}{\left(\Omega_{\alpha}-\omega_{1}\right)\left(\Omega_{\beta}-\omega_{2}-\omega_{3}\right)\left(\Omega_{\gamma}-\omega_{3}\right)}
$$

$$
\begin{aligned}
& \gamma_{I 7 I J}^{\eta j k l}\left(\omega_{1} \cdot \omega_{2} \cdot \omega_{3}\right)= \\
& -\sum_{\alpha \beta \gamma \gamma \delta} \frac{\left.V_{-\beta \gamma \delta} S_{\alpha \alpha} S_{\beta p} S_{\gamma} S_{\delta} \mu_{\alpha \beta \beta}^{i} \mu_{-\alpha}^{l} \mu_{-\gamma}^{k} \mu_{-\delta}^{l}\right)\left(\Omega_{\beta}-\omega_{-}-\omega_{3}\right)\left(\Omega_{\gamma}-\omega_{-}\right)\left(\Omega_{\delta}-\omega_{3}\right)}{}
\end{aligned}
$$

where the superscripts $i, j . k$ denote the index of components. $x, y z$ and the subscripts $\alpha, \beta, \gamma, \ldots$ represent the 
corresponding oscillators. The intensities of linear obsorption and two-photon absorption are proportional to immaginary parts of $\alpha(\omega)$ and $\chi-\omega . \omega-\omega, \omega)$. respectively. The orientationally averaged $\gamma$ can be obtained by

$$
\gamma=\left[3 \sum_{j} \gamma_{i j i j}-\sum_{i \neq j}\left(2 \gamma_{i j j j}-\gamma_{i j j i}\right)\right] / 15
$$

\section{Applications of CEO}

The electronic excitations of poly(p-phenylene vinylene) (PPV) oligomers and their scaling with molecular size have exanined using CEO method by Mukaniel and collaborators. ${ }^{172}$ They have examined the ground state density matrix in the atomic basis instead of orbital basis. Substituted conjugated molecules are known to have large nonlinear optical properties.

The electronic spectra of a family of unsubstituted and acceptor substituted carotenoids were investigated by Mukantel et $a f^{17,24,25}$ They have studied the interactions between bridge and the substituent as the bridge size increases. For

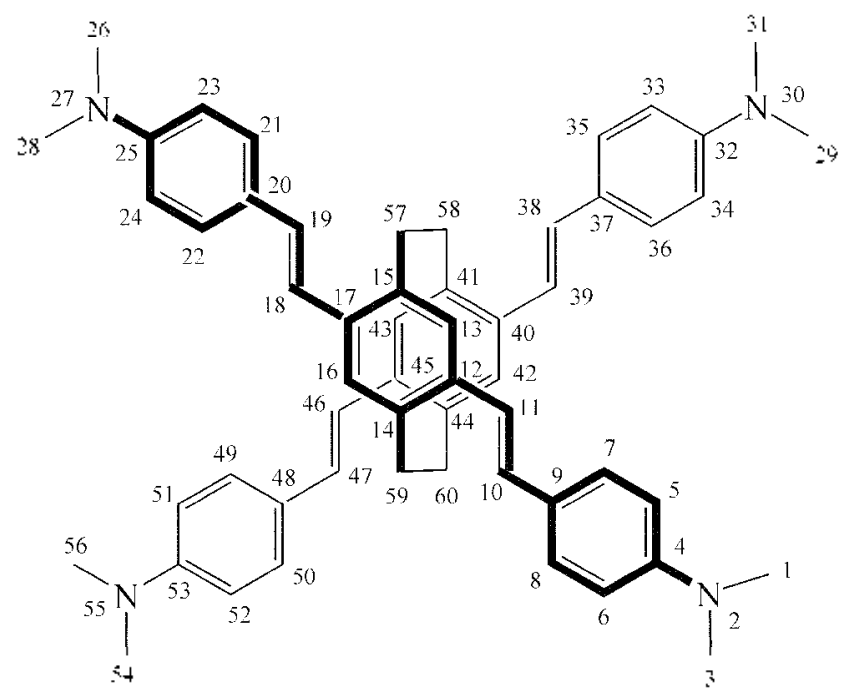

Figure 1. Donors-acceptors substituted tetrastyryl-[2,2]paracyclophane and its atomic numbering.

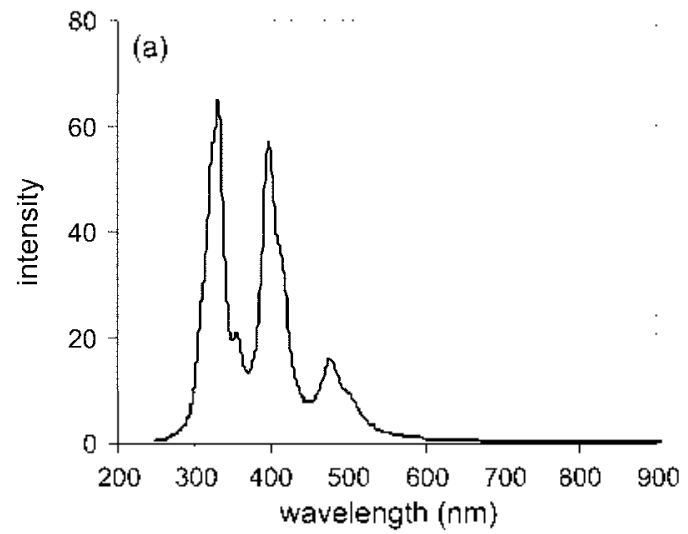

very long bridge the effects of the acceptor and the bridge regions can be separated. Optical properties of acceptorsubstituted molecules with shorter bridges can be attributed to quantum confinement. which is important when the bridge size becomes comparable to the coherence length.

We have extended the CEO formalisn to obtain the frequency-dependent two-photon absorption (TPA) spectra of a family of tetrastyryl-[2.2]paracyclophane derivatives with various through-space charge transfer configurations involving different combinations of donor and acceptor groups at the terminal styry $1^{3 ! 1}$ and the diagonal and non-diagonal tensor elements of linear quadratic and cubic polarizabilities of benzene and octatetraene. . $^{31}$

Figure 2 shows the linear absorption and TPA spectra of donor-acceptor substituted paracyclophane (see Figure 1 for molecule and its numbering). The linear absorption peaks correspond to the excited states which can be accessed by one photon with the corresponding transition energies. while TPA peaks correspond to the excited states which can be accessed by simultaneous two photons with half the corresponding transition energies. The linear absorption spectrum (Im $\left.\left(\alpha_{x x}+\alpha_{y}-\alpha_{s}\right)\right)$ is obtained with the line width of $0.08 \mathrm{eV}$. The linear absorption spectrum shows three peaks (LAl. LA2, and LA3 starting from the low energy).

The peaks around $480 \mathrm{~nm}$ (LAl) have slightly larger variation between molecules (though it is less than $4 \mathrm{um}$ ) than other peaks. The peaks around $330 \mathrm{~nm}$ (LA3) only show about 1 num variation. The lowest twenty modes have been used to compute TPA spectrum. The orientationally averaged TPA spectrum computed using diagonal and off-diagonal tensor elements $\left(\ln (\gamma), \gamma=\left[3 \sum_{j} \gamma_{1 m}-\sum_{i \neq j}\left(2 \gamma_{1 \eta, j}-\gamma_{\eta, j}\right)\right] / 15\right)$ with $0.08 \mathrm{eV}$ line width with units in $10^{-34} \mathrm{esu}$. It should be noted that the TPA states lie 0.3-0.9 eV above the first and second linear absorption peaks depending on the particular combination of donor and acceptor. This is similar to the linear quadrupolar șistems of bis-donor diphenylpolyene and bis-styrylbenzene derivatives 32 but in contrast to linear polyenes and several classes of $\alpha, \omega$-substituted polyenes

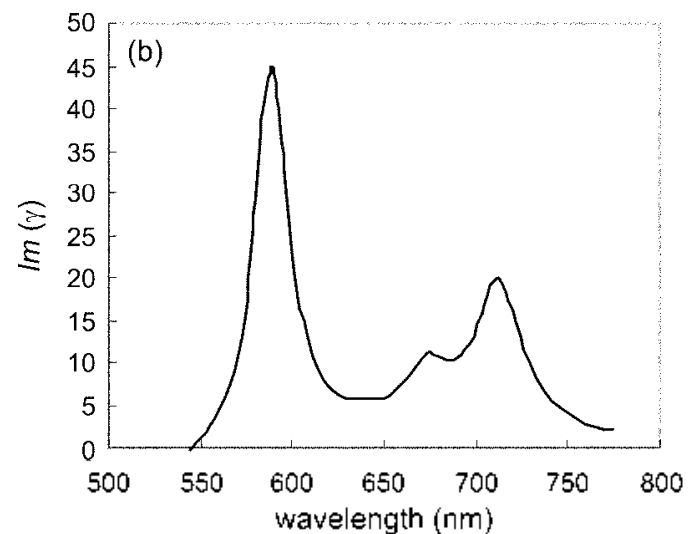

Figure 2. Linear absorption spectra $\left[\operatorname{Im}(\alpha(\omega))\right.$ in $10^{-233}$ esu $]$ (a) and orientationally averaged two-photon absorption spectra $[\operatorname{Im}(x,-\omega . \omega,-\omega$. (b)) in $10^{-3-1}$ esl] (b) of tetrastyry $1-[2,2]$ paracyclophane. 

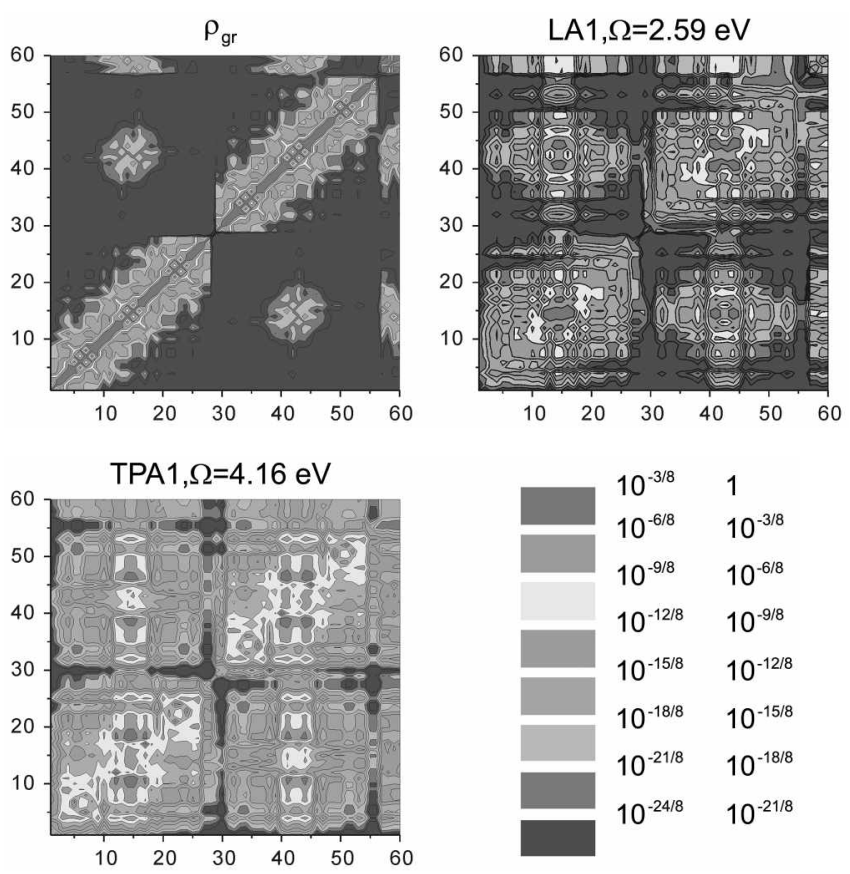

Figure 3. Contour plots of ground state density matrix $\left(\rho_{g}\right)$ and oscillators which are dominant for the lowest linear absorption peak (LA1) and the lowest TPA peak (TPA 1)

with more than two or three double bonds. where the onephoton allowed state lies at slightly higher energy than the lowest two-photon state. ${ }^{33.34}$

Figure 3 shows the ground state density matrix and the transition density matrices corresponding to the lowest linear absorption and TPA states. To represent the electron-hole coherences. the abscissa and ordinate represent the electron and hole axes. respectively. In ground state density matrix. the diagonal elements $\left(\rho_{m n}\right)$ represent the charges of the atom $n$. and the off-diagonal elecments $\left(\rho_{\text {tmm }}\right)$ is symmetric and represent the bond order between atoms $n$ and $m$. For LAl. the four electron donating dimethylamino groups push the electrons to the central cyclophane rings. making the electrons localize there. and indicating a strong coherence between the two central cyclophane rings by through-space interactions. The lowest and second lowest oscillators are responsible for this absorption peak. The $397 \mathrm{~nm}$ peak can be assigned by third and fourth oscillators which have similar modes to the first and second oscillators except that electrons are more diffused on the styryl groups and less localized than the first and second modes. This mode shows a somewhat weaker coherence between central cyclophane rings. The $330 \mathrm{~nm}$ absorption peak has coherence between central cyclophane rings and the terminal styryl groups as well as weakened through space $\pi-\pi$ coherence

In general. the higher order nonlinear optical processes reflect the interaction of electron and hole pairs between distant atoms. This can be seen in the transition density matrices corresponding to the lowest linear absorption and TPA peaks in Figure 3. All molecules show a large coherence length. in particular between the central cyclophane ring and the counter molecular units which is apart from the central cyclophane by through-space charge transfer interactions. The coherence can be seen only in one direction, in other words. the coherence is only in the lower triangular region of the contour map of the density matrix of the oscillator. This may be due to the fact that the two donors are in upper paradistyry lbenzene and two acceptors are in lower one connected by two ethylene linkers to make a cyclophane. In this case electron transfer is unidirectional. through-space. from the donor-substituted distyryl-benzene to the acceptor-substituted one for TPA1, and has the opposite direction for TPA2.

Very different TPA spectra even though the linear absorption is virtually identical are notified in various paracyclophane derivatives. ${ }^{3(1)}$ Through-space charge transfer interactions may thus be used to control and fine-tune the TPA frequencies and cross-sections. The observed trends are interpreted using the electronic density matrices corresponding to dominant oscillators in one- and two-photon absorption as well as the ground state. The lowest four oscillators are responsible for most of the TPA cross-sections and a simple effective threestate model cannot account for the relative TPA crosssection for the systems investigated. As another application of CEO. we obtained the coherence length of the time dependent densities to first $\left(\rho^{(1)}\right)$ and second order $\left(\rho^{(2)}\right)$ in the electric field obtained from the CEO method, and found that the coherence lengths are consistent with the sizes that give the maximum values of intramolecular charge transfer (ICT)-driven polarizability $\left(\alpha^{-\mathrm{T}}\right)$ and first hyperpolarizability $\left(\beta^{\mathrm{CT}}\right)^{\text {35 }}$

\section{Concluding Remarks}

Molecular eigenstates use a single-particle basis set. However an optical excitation moves an electron from an occupied orbital to an unoccupied orbital. thereby creating an electron-hole pair. Therefore. the natural description of the optical response should be based on the simultaneous and coupled dynamics of these electron-hole pairs. This is the main reason for the success of the CEO. From the CEO calculations. a real space analy'sis of linear absorption which explains the origin of each optical transition is possible by the electronic mode matrices graphically: As is often the case, only a few oscillators dominate the response. hence the theoretical description is much simplified. In atoms. collective excitations have been found to converge to local modes rather than to normal modes ${ }^{36 i}$ The electron-hole pairs are known to be loosely bound and form Wannier exciton in semiconductors. ${ }^{37}$ while tightly bound and can be considered as a single particle (Frenkel exciton) in molecular aggregates. ${ }^{3 \times 39}$ The oscillator approach allows us to develop a natural framework for the design of molecules with efficient nonlinear optical properties. We can explore how different regions of the molecule couple and affect each other.

Acknowledgment. This work was supported by KORDIC (4th Supercomputing Application Support Program). 


\section{References}

1. Brédas. J. L.: Comil. J.: Belionne. D.: Santos. D. A. dos: Shuai. Z. G. Acc. Chem. Res. 1999.32.267.

2. Rumi, M.: Ehrlich, J. E.: Heikal, A. A.: Perry. I. W.: Barlow S.: Hu, Z: McCord-Malughon, D: Parker, T. C: Rockel. H: Thavumanavan. S.: Marder. S. R.: Beljonne. D.: Brédas. I.-L. J. Am. Chem. Soc. 2000. 122.9500.

3. Kanis. D. R.: Ratner. M. A.: Marks. T. J. Chem. Res, 1994, 94. 195

4. Heeger. A. J. Ret Hod Plns. 2001. $73,681$.

5. Baldo, M. A.: Thompson. M. E; Forrest, S. R. Nature 2000, 103. 750

6. Frolov. S. V.: Gellermann. W. Ozaki. M.: Yoshino. K.: Vardeny. Z. V. Plws. Ren. Lett. 1997. 78. 729

7. Lalamam. S. J.: Garito. A. F. Plys. Rev A 1979. 20.1179

8. Docherty. V. J.: Pugh. D; Morley, I. J. Chem. Soc. Faraday Trans. 2 1985. 81. 1179 .

9. Kanis, D. R.: Ratner, M. A.: Marks, T. J.: Zemer. M. C. Chent. Mater. 1991.3. 19

10. Ward. I. F. Rev Mod Phns. $1965.37,1$

11. Dirk. C. W.: Kuzyk. M. G. Phs. Rev A 1989.39.1219.

12. Kuzyk. M. G.: Dirk. C. W. Phis. Rev. A 1990, H1. 5098.

13. Oudar. J. L. J. Them. Phis. 1977, 67.446.

14. Blanchard-Desce. M.: Barzoukas. M. J. Opt. Soc. Ant. B 1998. I5. 302

15. Marder, S. R.: Berattan. D. N.: Cheng. L. T. Science 1991. 252. 103.

16. (a) Zyss. J. J. Chem. Plys. 1979. 70. 3333: (b) Zyss. J. J. Chem. Phys. 1979. 70.3341: (c) Zyss. J. J. Chem. Phss. 1979.71. 909.

17. Mukamel, S: Tretiak. S.: Wagersreiter T.: Chernvak. V. Science $1997,277,781$

18. Mukamel, S: Takahashi, A. Wang. H. X: Chen. G. Science 1994. 266. 250.

19. Mukamel. S.: Wang. H. X. Phns. Rev Lett. 1992.69.65.

20. Toury. T.: Zyss. J.: Chernyak. V: Mukamel. S. J. Phys. Chem. A $2001,105,5692$.

21. Tretiak, S. Mukamel, S. Chent Rev. 2002. 102,3171 and references are therein.

22. Bazan. G. C.: Oldham. W. J.. Jr.: Lachicotte. R. J.: Tretiak. S.: Chernyak. V: Mukamel. S. J. Am. Chem. Soc. 1998. 120.
9188.

23. Zyss. J.: Ledoux. I.; Volkov. S.: Chemyak. V: Mukamel. S.: Bartholomew. G. P.: Bazan. G. C. J. Am. Chem. Soc. 2000. 122. 11956

24. Tretiak. S.: Chemyak. V.: Mukamel. S. J. Am. Chem. Soc. 1997. 119. 11408

25. Tretiak. S.; Chemyak. V.: Mukamel, S. Chent. Phns. Lett 1996. 259.55

26. (a) Saad. Y. Mumerical Methods for Large Eigmalue Problens: University Press: Manchester. 1992: (b) Chernyak. V: Schultz. M. F.: Mukamel. S.: Tretiak. S.: Tsiper. E. V. J. Chem. Phus. 2000. 113. 36 .

27. (a) Davidson. E. R. J. Comput. Phys. 1975, 17,87 ; (b) Stratmann E. R.: Scuseria, G. E.; Frisch. M. J. J. Chem. Phos 1998. 109. 8218.

28. Wiberg. K. B.: Stratmann. E. R.: Frisch. M. T. Chem. Phus. Lett 1998.297 .60

29. Tretiak. S.: Saxena, A.; Martin. R. L.; Bishop, A. R. J. Phw Chent. B 2000. 10+. 7029

30. Lee, J. Y; Tretiak. S.: Volkov. S.; Bazan, G. C.; Zyss. J.; Muliamel. S. submitted.

31. Lee. T. Y.: Tretiak. S.: Volkov. S.: Kim. K. S.: Mukamel. S. in preparation.

32. Albota. M.: Beljonne, D.; Brédas, J.-L; Ehrlich. J. E.; Fu. J.-Y; Keikal, A. A.: Hess. S. E.; Kogej, T.; Levin. M. D.: Marder. S. R. McCord-Maughon. D: Perry. J. W. Röckel. H; Rumi, M. Subramaniam. G.: Webb. W. W: Wu. X.-L.: Xu. C. Science 1998. 281.1653

33. Hudson. B. S.: Koller. B. E. Amm. Ren. Phys. Chem. 1974. 25 437.

34. Hudson. B. S.: Kohler, B. E; Schulten. K. In Excited States. Vol. 6. Lim, E. C., Ed.: Academic Press: New York. 1982: pp 1-95.

35. Lee. J. Y.: Mhin. B. T.: Mukiamel. S.: Kim. K. S. submitted.

36. Orr. B. J.: Ward. J. F. Mol. Plns. 1971. 20.513.

37. Klessinger. M.: Michl. I. Excited States and Photochemistry of Onganic Holecules: VCH: New York, 1995.

38. Nonlinear Optical Properties of Organic Molecules and Cnstals; Zyss. J.: Chemla, D. S., Eds.: Academic Press: Florida. 1987: Vol. 1 and 2.

39. Rodenberg. D. C.: Heflin. I. R.: Garito. A. F. Nattre 1992. 359. 309. 